\title{
Putative Targets as Vaccine Candidates with Respect to Biofilm Formation Procedure in Staphylococci
}

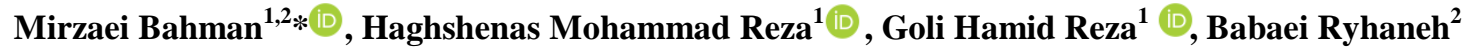 \\ ${ }^{1}$ Department of Medical Microbiology and Virology, Faculty of Medicine, Mazandaran University of Medical Sciences. \\ ${ }^{2}$ Department of Medical Microbiology and Virology, School of Medicine, Zanjan University of Medical Science.
}

\section{A R T IC LE IN F O}

\section{Mini Review Article}

VacRes, 2019

Vol. 6, No.2, 9-13

Received: February 25, 2020

Accepted:May 27, 2020

Pasteur Institute of Iran

*Corresponding Author: Mirzaei

Bahman. Department of Medical

Microbiology and Virology, Faculty of

Medicine, Mazandaran University of

Medical Sciences.

Department of Medical Microbiology and Virology, School of Medicine, Zanjan University of Medical Science.

Email: dr.bahman.m@gmail.com

Tel/Fax: +982433140345

KEYWORDS: Putative Vaccine, Biofilm Formation, Staphylococcus aureus, Staphylococcus epidermidis

\section{A B S T R A C T}

The amount of multidrug-resistant (MDR) strains, especially methicillin-resistant Staphylococcus aureus and Staphylococcus epidermidis, as frequent causes of nosocomial and device-related infections have increased. Biofilm formation is an essential requisite in staphylococcal pathogenicity. It is considered as a bacterial surveillance, antibiotic resistance, and transition of antibiotic resistance genes factor. Therefore, biofilm-related macromolecules have been suggested as putative new vaccine candidates to combat staphylococcal infections. Based on the MEDLINE and Google scholar databases, some Staphylococci macromolecules are involved in the biofilm formation process and have been reviewed as putative vaccines. Based on experiments, common staphylococcus antigens could prevent the progress of the caused diseases by this genus. Moreover, considering related stages in biofilm formation, a multivalent putative vaccine (protein and polysaccharide) candidate could be enhancing the eradication chance of aforementioned bacterial families.

\section{Citation:}

Mirzaei B, Haghshenas M R, Goli H, Babaei R. Putative Targets as Vaccine Candidates with Respect to Biofilm Formation Procedure in Staphylococci. vacres. 2019; 6 (2) :9-13. DOI: 10.29252/vacres.6.2.9

\section{INTRODUCTION}

Staphylococcaceae family is non-motile, non-sporeforming, catalase-negative bacteria that grow on most bacteriological culture media at both anaerobic and aerobic conditions. Based on the ability of bacteria to produce coagulase, a protein enzyme that facilitates the conversion of fibrinogen to fibrin, staphylococci are divided into coagulasepositive and -negative Staphylococcus (CoPS and CoNS, respectively [1]. Unlike the CoNS, the CoPS often have golden discoid colonies surrounded by a zone of $\beta$-hemolysin. Because of increasing cases of antibiotic-resistant patterns in the strains of staphylococci, there is a demand for an effective prophylactic vaccine against these bacteria. Staphylococcaceae family is considered as the etiological agents of several mild and intense disorders, such as sepsis and endocarditis [1]. Based on the preclinical models, various antigens can prevent the spread of staphylococcal diseases either alone or in a combination with other antigens. The development of a protective vaccine to cease the spread of sepsis is one of the most challenging issues in pharmacology research [1].

$S$. aureus is a primary pathogen causing a wide range of diseases, such as mild skin and soft tissue infections, bacteremia, endocarditis, pneumonia, metastatic infections, sepsis and toxic shock syndrome in hospitalized patients. The reason for this wide range of symptoms might be related to undiscovered factors that make the host susceptible to colonization [2]. The contamination of medical devices with $S$. aureus inserted into the patient's body might be remarkably dependent on the patients' health. There are similarities in the developed infections caused by staphylococci biofilm and usually highly intensive care is needed in such cases. Infections caused by $S$. epidermidis are more difficult to treat by antibiotic therapy in comparison with S. aureus [3]. Moreover, medical devices act as a spreading source of several bacterial infections to different parts of the human body. Over the past decades, there has been an increase in the nosocomial infections caused by staphylococcus species, especially $S$. aureus $[4,5]$. Since the 1960 s, the first methicillin-resistant $S$. aureus (MRSA) strains were detected which have remained a major global challenge [6]. Thus from the molecular pathogenesis perspective, it is essential to know the relevant factors involved in such biofilm formations and to discover their physiological status within the body.

S. epidermidis is an inhabitant of human skin. For a long time, it was only considered as a contaminant when cultured from blood or tissue samples $[7,8]$. Since $S$. epidermidis is a part of normal skin flora, it probably initiates contamination after implantation of a medical device. In recent years, $S$. epidermidis has been accepted as a leading cause of nosocomial 
bloodstream infections, especially in patients with prosthetic medical devices [9, 8]. S. epidermidis is an opportunistic pathogen, principally known as the cause of infection in immunocompromised patients [10]. Biofilm formation of $S$. epidermidis is a critical factor in the pathogenesis because it can be colonized on medical devices which makes it resistant to multiple antibiotics and host defenses. There is an essential need to remove or replace the biofilm contaminated medical implants. Moreover, studies are needed to be done to provide new and effective vaccines against staphylococcal biofilm formation [11]

CoNS which inhabit on a person's skin include S. hominis, S. epidermidis, saprophyticus, $S$. warneri, $S$. cohnii, $S$. saccharolyticus, S. haemolyticus, S. capitis and S. lugdenensis. They are normally harmless to their host. Most staphylococci including $S$. epidermidis in the cases that the skin is injured might be pathogenic. CoNS colonization seems to be relevant to the specific sites of the infection and its abundance. For instance, S. saprophyticus which is a common inhabitant of inguinal and perineal areas, is an etiological agent of urinary tract infections [12].

Biofilm formation and consistency of host immune evasion of $S$. epidermidis and $S$. aureus make them the main concern of the nosocomial infections in hospitals [13, 14]. Despite being a part of human flora, the ability to adhere to the medical device surfaces and developing multilayered structures, known as "biofilm", makes them problematic [15]. Biofilm is defined as a community of cells encased within an exopolymeric matrix and attached to a surface. It has been proved that biofilms are resistant to antimicrobial therapy and host defense [13].

Many studies have demonstrated that biofilm developed in a 2step physiologically process; primary adherence of the cells to the site and the maturation of the biofilm. Phase-specific factors are needed for each of these steps. In general, there is no agreement about different steps of biofilm formation in staphylococci. We review here three main stages, namely attachment, maturation/aggregation and detachment [15].

\section{1- Attachment}

The first stage of biofilm formation is attachment. That is, bacteria attach to their host cell membrane by bacterial appendages which are cell-surface components that facilitate adhesion to other cells. Matrix proteins play a critical role in both adherence and the evasion of the host immune system. This makes matrix proteins as important virulence factors in Staphylococci. The Gram-positive bacterial proteins are divided into two families; microbial surface components, recognizing adhesive matrix molecules (MSCRAMMs), and serine-rich repeat proteins (SRRPs) [16].

One of the most important factors of colonization is the interaction between the matrix proteins of the host and MSCRAMMS. A set of MSCRAMMs with a capacity to link to protein matrix in humans, such as fibrinogen, fibronectin, and several matrix proteins are synthesized by $S$. epidermidis and $S$. aureus [17]. The common structure of MSCRAMMs consists of an exposed ligand-binding domain, a membrane-spanning domain (mostly with a repeated structure) and a domain responsible for the covalent and non-covalent attachment to the bacterial surface. Sortases are a family of prokaryotic enzymes that catalyze the covalent attachment of the MSCRAMMS LPXTG (Leu-Pro-any-Thr-Gly) motif, which is split between the threonine and glycine residue [18]. Sortases anchor up to 21 and 12 different LPXTG proteins to the cell wall in $S$. aureus and $S$. epidermidis, respectively [19, 20].
MSCRAMMs can mediate indirect binding to host plasma-covered surfaces with fibronectin $(\mathrm{Fn})$, collagen $(\mathrm{Cn})$ and fibrinogen $(\mathrm{Fg})$ as matrix proteins. Cell surfaces are covered with a different macromolecules, such as proteins including Embp, GehD, SdrG, SdrF, AtlE and Aae autolysins as well as polysaccharides (i.e. cell wall teichoic acid (TA) and polysaccharide intercellular adhesion; PIA) and matrix-binding determinants [21, 22]. Serine-aspartate repeat (Sdr) protein family members are categorized into two distinct species; however, their function is the same [23]. Both species use autolysin $\mathrm{AaP}$ proteins to form their noncovalent bonds, maintaining the three-dimensional structures of the macromolecules [24]. Autolysins are the most frequent proteins on staphylococcal cell surfaces, non-covalently linked to teichoic acid [25]. These enzymes have a considerable role in the rate of cell wall- turnover and are critically important for the bacterial attachment. Moreover, they facilitate the attachment on plastic surfaces and harbor binding sites for human matrix proteins [26]. The GehD lipase plays a more important catalytic role than the autolysins and it has an additional adhesive function [27]. Given attachment is the first step of biofilm formation, any of the surface-located macromolecules could be considered as a putative vaccine candidate [7].

\section{2- Maturation/Aggregation}

The maturation phase has two main characteristics in the biofilm formation; A) intercellular aggregation by a wide range of molecules including sticky macromolecules; B) formation of the three-dimensional structure of mature biofilm.

\section{Adhesive Forces}

Poly-N-acetylglucosamine (PNAG) is the most important PIA because its chemical composition is the most responsible molecule for adhesion in the Staphylococcal aggregation [28]. The extracellular matrix of staphylococcal biofilm is often called "slime" which is consisted of several polymers including PIA, proteins and teichoic acids. The core polymer of PIA has a $\beta$-1, 6-linked $\mathrm{N}$-acetylglucosamine structure [29]. Homologs of PIA have been recently found in different biofilms of pathogens, which suggest its broad function in biofilm formation and biofilm-associated infections. PIA biosynthesis depends on the expression of the icaADBC operon. The expression of icaADBC is regulated by an array of environmental factors and regulatory proteins [30, 31]. The Intercellular Adhesion (ica) locus contains an Nacetylglucosamine (GlcNAc), a PIA deacetylase (icaB), a putative PIA exporter (icaC) and a regulatory gene (icaR) [32, 33]. Some strains without the ica genes have been isolated from biofilm-associated infection which suggests that PIA is not generally essential for biofilm formation in staphylococci [34, 35]. The proteinaceous intercellular adhesion is involved in cell accumulation of those strains that do not produce PIA polymer [7]. Accumulation-associated protein (Aap), is the most important protein involved in PIA-independent biofilm formation and contains various domains including domain A, linked to corneocytes, making it of great importance for skin colonization [36]. To induce biofilm formation, Aap interacts with PIA, and then a $220 \mathrm{kDa}$ Aap protein needs to be proteolytically broken down to a smaller $140 \mathrm{kDa}$ form [37, 38]. The function of the staphylococcal surface proteins, SSP-1 and SSP-2, might be similar to Aap role in terms of biofilm production [39]. S. epidermidis surface (Ses) proteins have been proven to be formed by SSPs; therefore, providing cell-cell adhesion over longer distances which explains how these 
proteins contribute to the aggregation step of the biofilm development.

Considering the PIA-independent biofilm formation, other involved proteins are biofilm-associated proteins (Bap) and biofilm-associated homolog proteins (Bhp) [7]. Bap family might be essential in biofilm production because of the presence of Bap homologs in other bacteria and the vital role of this large protein in $S$. epidermidis derived from mastitis [40, 41]. Recent studies have identified that extracellular matrix binding protein (Embp) and fibronectin-binding MSCRAMM facilitate biofilm formation as a proteinaceous intercellular adhessive [42]. Many Gram-positive bacteria have TA polymers, such as $S$. aureus and $S$. epidermidis. There are two sorts of TA, namely cell wall-linked TA (WTA) and lipoteichoic acid (LTA) which is linked to the cell wall by a lipid anchor [43, 44]. TA has a polyanionic character and has been described as a stabilizing factor [7]. D-alanylation of TA in $S$. aureus is a vital factor in biofilm formation [45]. Moreover, a probable role for TA in S. epidermidis virulence could be its attachment to the fibronectin-coated surfaces [46].

\section{Biofilm Structure Disrupting Force}

When the biofilm matures, a specific 3D structure is formed through the fluid-filled channels [47]. Based on the findings, modulin proteins as quorum-sensing (QS) mediators play a key role in the mechanisms leading to the channel formation and biofilm structures [7]. Phenol-soluble modulins (PSMs) are a class of surfactant-like peptides, mainly assigned as pro-inflammatory molecules in S. epidermidis. They are subdivided by an amphipathic alpha-helical structure into two classes: the shorter type is called $\alpha$ type, which has a length of approximately 20 amino acids (PSM $\alpha, \gamma, \delta$, and $\varepsilon$ ) and the longer type that is called $\beta$ type with a length of approximately 40 amino acids (PSM $\beta$ s) [7]. Shifting $\beta$-type and PSMs in PSM expression have been observed when biofilm constructed. In other hand, the expression of PSMs likely constitutes a key factor contributing to the switch between an aggressive and a silent form of $S$. epidermidis physiology during the infection. Detachment of biofilms, dissemination of pathogen and the attraction of immune cells are related to PSMs expression. Whereas suppression of the production of PSMs in the biofilm stage enables the cells to stick together and to evade the host immune defense. [48]. The development of biofilm in $S$. epidermidis is directly related to the down- and up-regulation of PSM expression [7]. At a lower concentration, the PSM $\beta$ s might form "holes" in an early biofilm and lead to the formation of spaces and channels in the biofilm structures [7, 49].

\section{3- Detachment}

Disperse of bacteria to connect to another colonization site during the establishment of mature biofilm in staphylococci is known as detachment. It may happen by either detachment of single cells or larger cell aggregates. Cell dispersal not only leads to embolism, sepsis and hospital-acquired pneumonia it also leads to biofilm formation at other sites [50]. In staphylococci, agr QS system controls factors that will change the biofilm surface when the rate of associated factors is relatively high [51]. The increase of PSM $\beta$ leads to cluster detachment of the biofilm. As long as the biofilm matures, it results in a systemic spread of its fragments [49]. It has also been suggested that PSM $\gamma$ (identical to $\delta$-toxin) acts as a cellcell disruptive factor [52].

The Biofilm-Based Putative Vaccine Candidates
Biofilm formation is a clinical challenge. It increases the antibiotic resistance patterns and bacterial evasion from the host defense [14]. Biofilm formation has great importance in a wide range of infections and has been accepted as a bacterial mode of growth. According to the National Institutes of Health (NIH), approximately $80 \%$ of human biofilm-related infections are common [13]. Medical device-associated infections caused by biofilm formation of $S$. epidermidis and $S$. aureus have led to challenging and complicated medical processes. The emergence of antibiotic-resistant strains of staphylococci, mainly MRSA, emphasizes this matter [19].

So far, several bacterial surface-located components including serine-aspartate repeat protein $G(\mathrm{SdrG})$, serineaspartate repeat-protein $\mathrm{F}$ (sdrF), clumping factor A (ClfA), GehD lipase and extracellular matrix-binding protein (Embp) which are engaged in the initial phase of biofilm production as well as autolysin E (AtlE) have been evaluated as putative staphylococcal vaccine candidates [53- 56]. Furthermore, the MSCRAMMs/surface proteins have also been considered in this regard [53- 56].

In conclusion, vaccine development against staphylococcal infections is still in its infancy. As it was previously mentioned, biofilm has resistance against antibiotics and could escape from the host immune system. Recently, several studies have been accomplished based on the selection of antigens to eradicate the biofilm-related infections. General immunization along with using short-term medical implants such as venous catheters seems to be more cost-efficient than removing and replacing the contaminated devices. For permanent medical device users, removing the contaminated device might be risky because of the long hospitalization time and increase in healthcare costs. Thus, justifiable and cost-effective methods must be considered.

\section{ACKNOWLEDGMENT}

The authors wish to acknowledge the Zanjan University of Medical sciences for funding. The authors are also grateful for the support of colleagues at Microbiology Department of the Pasteur Institute of Iran.

\section{CONFLICT OF INTEREST}

The authors declare that they have no conflict of interest.

\section{REFERENCES}

1. Colditz GA, Brewer TF, Berkey CS, Wilson ME, Burdick E, Fineberg $\mathrm{HV}$, Mosteller F. Efficacy of BCG vaccine in the prevention of tuberculosis: meta-analysis of the published literature. Jama. 1994 Mar 2;271(9):698-702.

2. van Belkum A. Staphylococcal colonization and infection: homeostasis versus disbalance of human (innate) immunity and bacterial virulence. Current opinion in infectious diseases. 2006;19(4):339-44. doi:10.1097/01.qco.0000235159.40184.61.

3. Otto M. Staphylococcus epidermidis - the'accidental'pathogen. Nature reviews microbiology. 2009 Aug;7(8):555-67.

4. Dryden MS. Skin and soft tissue infection: microbiology and epidemiology. International journal of antimicrobial agents. $2009 \mathrm{Jul}$ $1 ; 34:$ S2-7.

5. Hogea C, Van Effelterre T, Cassidy A. A model-based analysis: what potential could there be for a $S$. aureus vaccine in a hospital setting on top of other preventative measures? BMC infectious diseases. 2014;14:291 doi:10.1186/1471-2334-14-291.

6. Adhikari RP, Karauzum H, Sarwar J, Abaandou L, Mahmoudieh M, Boroun AR et al. Novel structurally designed vaccine for S. aureus alpha- 
hemolysin: protection against bacteremia and pneumonia. PloS one. 2012;7(6):e38567. doi:10.1371/journal.pone.0038567.

7. Van Mellaert L, Shahrooei M, Hofmans D, Eldere JV. Immunoprophylaxis and immunotherapy of Staphylococcus epidermidis infections: challenges and prospects. Expert review of vaccines. 2012;11(3):319-34. doi:10.1586/erv.11.190.

8. Sellman BR, Howell AP, Kelly-Boyd C, Baker SM. Identification of immunogenic and serum binding proteins of Staphylococcus epidermidis. Infection and immunity. 2005;73(10):6591-600. doi:10.1128/iai.73.10.6591-6600.2005.

9. Fattom A, Shepherd S, Karakawa W. Capsular polysaccharide serotyping scheme for Staphylococcus epidermidis. Journal of clinical microbiology. 1992;30(12):3270-3.

10. Otto M. Bacterial evasion of antimicrobial peptides by biofilm formation. Current topics in microbiology and immunology. 2006;306:2518 .

11. Hu J, Xu T, Zhu T, Lou Q, Wang X, Wu Y et al. Monoclonal antibodies against accumulation-associated protein affect EPS biosynthesis and enhance bacterial accumulation of Staphylococcus epidermidis. PloS one. 2011;6(6):e20918. doi:10.1371/journal.pone.0020918.

12. Kloos WE, Schleifer KH. Simplified scheme for routine identification of human Staphylococcus species. Journal of clinical microbiology. 1975;1(1):82-8.

13. Paharik AE, Horswill AR. The Staphylococcal Biofilm: Adhesins, Regulation, and Host Response. Microbiology spectrum. 2016;4(2). doi:10.1128/microbiolspec.VMBF-0022-2015.

14. Kiedrowski MR, Horswill AR. New approaches for treating staphylococcal biofilm infections. Annals of the New York Academy of Sciences. 2011;1241:104-21. doi:10.1111/j.1749-6632.2011.06281.x.

15. Kostakioti M, Hadjifrangiskou M, Hultgren SJ. Bacterial biofilms: development, dispersal, and therapeutic strategies in the dawn of the postantibiotic era. Cold Spring Harbor perspectives in medicine. $2013 \mathrm{Apr}$ 1;3(4):a010306.

16. Arora S, Uhlemann AC, Lowy FD, Hook M. A Novel MSCRAMM Subfamily in Coagulase Negative Staphylococcal Species. Frontiers in microbiology. 2016;7:540. doi:10.3389/fmicb.2016.00540.

17. Patti JM, Allen BL, McGavin MJ, Hook M. MSCRAMM-mediated adherence of microorganisms to host tissues. Annual review of microbiology.

doi:10.1146/annurev.mi.48.100194.003101.

18. Marraffini LA, Dedent AC, Schneewind O. Sortases and the art of anchoring proteins to the envelopes of gram-positive bacteria. Microbiology and molecular biology reviews : MMBR. 2006;70(1):192-221. doi:10.1128/mmbr.70.1.192-221.2006.

19. O'Neill E, Pozzi C, Houston P, Humphreys H, Robinson DA, Loughman A et al. A novel Staphylococcus aureus biofilm phenotype mediated by the fibronectin-binding proteins, FnBPA and FnBPB. Journal of bacteriology. 2008;190(11):3835-50.

20. Gill SR, Fouts DE, Archer GL, Mongodin EF, DeBoy RT, Ravel J et al. Insights on evolution of virulence and resistance from the complete genome analysis of an early methicillin-resistant Staphylococcus aureus strain and a biofilm-producing methicillin-resistant Staphylococcus epidermidis strain. Journal of bacteriology. 2005;187(7):2426-38.

21. Otto M. Staphylococcus epidermidis--the 'accidental' pathogen. Nature reviews Microbiology. 2009;7(8):555-67. doi:10.1038/nrmicro2182.

22. Heilmann C. Adhesion mechanisms of staphylococci. Advances in experimental medicine and biology. 2011;715:105-23. doi:10.1007/978-94007-0940-9_7.

23. Otto M. Staphylococcal biofilms. Bacterial biofilms. Springer; 2008. p. 207-28.

24. Schaeffer CR, Woods KM, Longo GM, Kiedrowski MR, Paharik AE, Büttner H, Christner M, Boissy RJ, Horswill AR, Rohde H, Fey PD. Accumulation-associated protein enhances Staphylococcus epidermidis biofilm formation under dynamic conditions and is required for infection in a rat catheter model. Infection and immunity. 2015 Jan 1;83(1):214-26.

25. Peschel A, Vuong C, Otto M, Götz F. The d-Alanine Residues ofStaphylococcus aureus Teichoic Acids Alter the Susceptibility to Vancomycin and the Activity of Autolytic Enzymes. Antimicrobial agents and chemotherapy. 2000;44(10):2845-7.

26. Heilmann C, Thumm G, Chhatwal GS, Hartleib J, Uekötter A, Peters G. Identification and characterization of a novel autolysin (Aae) with adhesive properties from Staphylococcus epidermidis. Microbiology (Reading, England). 2003;149(10):2769-78.

27. Bowden MG, Visai L, Longshaw CM, Holland KT, Speziale P, Hook M. Is the GehD lipase from Staphylococcus epidermidis a collagen binding adhesin? The Journal of biological chemistry. 2002;277(45):43017-23. doi:10.1074/jbc.M207921200.

28. Mack D, Fischer W, Krokotsch A, Leopold K, Hartmann R, Egge H et al. The intercellular adhesin involved in biofilm accumulation of Staphylococcus epidermidis is a linear beta-1,6-linked glucosaminoglycan: purification and structural analysis. J Bacteriol. 1996;178(1):175-83.

29. Gotz F. Staphylococcus and biofilms. Molecular microbiology. 2002;43(6):1367-78.

30. Mack D, Davies AP, Harris LG, Knobloch JK, Rohde H Staphylococcus epidermidis Biofilms: Functional Molecules, Relation to Virulence, and Vaccine Potential. Topics in current chemistry. 2009;288:157-82. doi:10.1007/128_2008_19.

31. Rohde H, Frankenberger S, Zahringer U, Mack D. Structure, function and contribution of polysaccharide intercellular adhesin (PIA) to Staphylococcus epidermidis biofilm formation and pathogenesis of biomaterial-associated infections. European journal of cell biology. 2010;89(1):103-11. doi:10.1016/j.ejcb.2009.10.005.

32. Vuong C, Voyich JM, Fischer ER, Braughton KR, Whitney AR, DeLeo FR et al. Polysaccharide intercellular adhesin (PIA) protects Staphylococcus epidermidis against major components of the human innate immune system. Cellular microbiology. 2004;6(3):269-75.

33. Heilmann C, Schweitzer O, Gerke C, Vanittanakom N, Mack D, Gotz F. Molecular basis of intercellular adhesion in the biofilm-forming Staphylococcus epidermidis. Molecular microbiology. 1996;20(5):1083-91. 34. Rohde H, Burdelski C, Bartscht K, Hussain M, Buck F, Horstkotte MA et al. Induction of Staphylococcus epidermidis biofilm formation via proteolytic processing of the accumulation-associated protein by staphylococcal and host proteases. Molecular microbiology. 2005;55(6):1883-95. doi:10.1111/j.1365-2958.2005.04515.x.

35. Arciola CR, Campoccia D, Baldassarri L, Donati ME, Pirini V, Gamberini $S$ et al. Detection of biofilm formation in Staphylococcus epidermidis from implant infections. Comparison of a PCR-method that recognizes the presence of ica genes with two classic phenotypic methods. Journal of biomedical materials research Part A. 2006;76(2):425-30. doi:10.1002/jbm.a.30552.

36. Macintosh RL, Brittan JL, Bhattacharya R, Jenkinson HF, Derrick J, Upton $\mathrm{M}$ et al. The terminal A domain of the fibrillar accumulationassociated protein (Aap) of Staphylococcus epidermidis mediates adhesion to human corneocytes. J Bacteriol. 2009;191(22):7007-16. doi:10.1128/jb.00764-09.

37. Hussain M, Herrmann M, von Eiff C, Perdreau-Remington F, Peters G. A 140-kilodalton extracellular protein is essential for the accumulation of Staphylococcus epidermidis strains on surfaces. Infection and immunity. 1997;65(2):519-24.

38. Rohde H, Burandt EC, Siemssen N, Frommelt L, Burdelski C, Wurster $\mathrm{S}$ et al. Polysaccharide intercellular adhesin or protein factors in biofilm accumulation of Staphylococcus epidermidis and Staphylococcus aureus isolated from prosthetic hip and knee joint infections. Biomaterials. 2007;28(9):1711-20. doi:10.1016/j.biomaterials.2006.11.046.

39. Veenstra GJ, Cremers FF, van Dijk H, Fleer A. Ultrastructural organization and regulation of a biomaterial adhesin of Staphylococcus epidermidis. J Bacteriol. 1996;178(2):537-41.

40. Lasa I, Penades JR. Bap: a family of surface proteins involved in biofilm formation. Research in microbiology. 2006;157(2):99-107. doi:10.1016/j.resmic.2005.11.003.

41. Latasa C, Roux A, Toledo-Arana A, Ghigo JM, Gamazo C, Penades JR et al. BapA, a large secreted protein required for biofilm formation and host colonization of Salmonella enterica serovar Enteritidis. Molecular microbiology. 2005;58(5):1322-39. doi:10.1111/j.1365-2958.2005.04907.x. 42. Christner M, Franke GC, Schommer NN, Wendt U, Wegert K, Pehle P et al. The giant extracellular matrix-binding protein of Staphylococcus epidermidis mediates biofilm accumulation and attachment to fibronectin. Molecular microbiology. 2010;75(1):187-207. doi:10.1111/j.13652958.2009.06981.x.

43. Hussain M, Wilcox MH, White PJ. The slime of coagulase-negative staphylococci: biochemistry and relation to adherence. FEMS microbiology reviews. 1993;10(3-4):191-207.

44. Hussain M, Hastings JG, White PJ. Comparison of cell-wall teichoic acid with high-molecular-weight extracellular slime material from Staphylococcus epidermidis. Journal of medical microbiology. 1992;37(6):368-75. doi:10.1099/00222615-37-6-368.

45. Otto M. Staphylococcal biofilms. Gram-Positive Pathogens. 2019 Oct $1: 699-711$.

46. Hussain M, Heilmann C, Peters G, Herrmann M. Teichoic acid enhances adhesion of Staphylococcus epidermidis to immobilized 
fibronectin. Microbial pathogenesis. 2001;31(6):261-70. doi:10.1006/mpat.2001.0469.

47. Costerton JW, Lewandowski Z, Caldwell DE, Korber DR, Lappin-Scott HM. Microbial biofilms. Annual review of microbiology. 1995;49:711-45. doi:10.1146/annurev.mi.49.100195.003431.

48. Yao Y, Sturdevant DE, Otto M. Genomewide analysis of gene expression in Staphylococcus epidermidis biofilms: insights into the pathophysiology of S. epidermidis biofilms and the role of phenol-soluble modulins in formation of biofilms. The Journal of infectious diseases. 2005;191(2):289-98. doi:10.1086/426945.

49. Wang R, Khan BA, Cheung GY, Bach TH, Jameson-Lee M, Kong KF et al. Staphylococcus epidermidis surfactant peptides promote biofilm maturation and dissemination of biofilm-associated infection in mice. The Journal of clinical investigation. 2011;121(1):238-48. doi:10.1172/jci42520. 50. Boles BR, Horswill AR. Staphylococcal biofilm disassembly. Trends in microbiology. 2011;19(9):449-55. doi:10.1016/j.tim.2011.06.004

51. Kong KF, Vuong C, Otto M. Staphylococcus quorum sensing in biofilm formation and infection. International journal of medical microbiology : IJMM. 2006;296(2-3):133-9. doi:10.1016/j.ijmm.2006.01.042.:164-9.
52. Otto M. Phenol-soluble modulins. International Journal of Medical Microbiology. 2014 Mar 1;304(2)

53. Bowden MG, Heuck AP, Ponnuraj K, Kolosova E, Choe D, Gurusiddappa $\mathrm{S}$ et al. Evidence for the "dock, lock, and latch" ligand binding mechanism of the staphylococcal microbial surface component recognizing adhesive matrix molecules (MSCRAMM) SdrG. The Journal of biological chemistry. 2008;283(1):638-47. doi:10.1074/jbc.M706252200.

54. Arrecubieta C, Lee MH, Macey A, Foster TJ, Lowy FD. SdrF, a Staphylococcus epidermidis surface protein, binds type I collagen. The Journal of biological chemistry. 2007;282(26):18767-76. doi:10.1074/jbc.M610940200.

55. Longshaw CM, Farrell AM, Wright JD, Holland KT. Identification of a second lipase gene, gehD, in Staphylococcus epidermidis: comparison of sequence with those of other staphylococcal lipases. Microbiology (Reading, England). 2000;146 ( Pt 6):1419-27. doi:10.1099/00221287-1466-1419.

56. Shahrooei M, Hira V, Stijlemans B, Merckx R, Hermans PW, Van Eldere J. Inhibition of Staphylococcus epidermidis biofilm formation by rabbit polyclonal antibodies against the $\mathrm{SesC}$ protein. Infection and immunity.

doi:10.1128/iai.01464-08 